\title{
Enhancing solubility and antibacterial activity using multi- component crystals of trimethoprim and malic acid
}

\author{
Yori Yuliandra ${ }^{1}$, Limson Jaya Hutabarat ${ }^{2}$, Resty Ardila ${ }^{2}$, Maria Dona Octavia ${ }^{2}$, and Erizal Zaini ${ }^{1}$ iD \\ ${ }^{1}$ Faculty of Pharmacy, Andalas University, Padang, Indonesia \\ ${ }^{2}$ School of Pharmaceutical Sciences Padang (STIFARM), Indonesia
}

\author{
Keywords \\ Antibacterial activity \\ Malic acid \\ Multicomponent crystals \\ Solubility \\ Trimethoprim
}

\author{
Correspondence \\ Prof Dr Erizal Zaini \\ Faculty of Pharmacy \\ Andalas University \\ Campus of Limau Manis \\ Pauh, Padang \\ West Sumatra 25163 \\ Indonesia \\ erizal@phar.unand.ac.id
}

\begin{abstract}
Aim: To improve the solubility and antibacterial activity of trimethoprim (TMP) by preparing its multicomponent crystals with malic acid (MA). Methods: Multicomponent crystals of TMP-MA were prepared by solvent co-evaporation. The solid-state properties were characterised by powder X-ray diffraction (PXRD), differential thermal analysis (DTA), Fourier transform infrared (FT-IR) spectroscopy, and scanning electron microscopy (SEM) analyses. The solubility was investigated in an aqueous medium, while the antibacterial activity against Escherichia coli was investigated using the agar disk diffusion method. Results: The PXRD pattern of the TMP-MA binary system differed from the starting materials, supporting the formation of a new crystalline phase (equimolar ratio). The DTA thermogram showed a single, sharp, endothermic peak at 212.5 ${ }^{\circ} \mathrm{C}$ attributable to the TMP-MA multicomponent crystal's melting point. FT-IR spectroscopy showed a solid-state interaction involving proton transfer between TMP and MA. The multicomponent crystal displayed a 2.5 -fold higher solubility and had increased antibacterial activity compared to TMP alone. Conclusions: The TMP-MA binary system forms salt-type multicomponent crystals that significantly increase solubility and antibacterial activity. Multicomponent crystal formation is a viable technique for modifying the physicochemical properties of active pharmaceutical ingredients.
\end{abstract}

\section{Introduction}

Trimethoprim (TMP) (Figure 1A), a 5-substituted-2,4diaminopyrimidine, is a well-known antibiotic that functions via selective inhibition of bacterial dihydrofolate reductase. TMP is used to treat urinary tract infections caused by various gram-positive and gram-negative bacteria (Hawser et al., 2006). Due to poor aqueous solubility, the drug has been designated as class II and low solubility-high permeability by the Biopharmaceutical Classification System (Li et al., 2005). Poor water solubility results in incomplete drug absorption in the gastrointestinal fluid, reducing the drug's pharmacological effectiveness (Kawabata et al., 2011). Previous studies have sought to enhance the solubility of TMP through various methods, including through the formation of solid dispersions, inclusion complexes and spherical crystallisation (Guptat et al., 1991; Pawar et al., 1998; Li et al., 2005).

The solid-state properties of biologically active compounds significantly influence their physicochemical, mechanical, and pharmacological effectiveness (Sheth \& Grant, 2005; Byrn \& Henck, 2012). The formation of a multi-component crystal phase is a promising technique to improve solid-state properties and thereby enhance solubility and dissolution rate (Dwichandra Putra et al., 2016; Zaini et al., 2019). The multi-component crystal phase is a unique crystalline phase containing more than one component, such as active pharmaceutical ingredients and complementary molecules (e.g. solvents or pharmaceutical excipients), arranged stoichiometrically. The molecules in the crystal lattice are bonded by non-covalent intermolecular interactions, such as hydrogen bonding 
and van der Waals forces. Multi-component crystals of pharmaceutical materials include cocrystals, salts, hydrates, and solvates (Cherukuvada et al., 2016; Berry \& Steed, 2017).

Incorporating active pharmaceutical ingredients into multi-component crystals has been shown to enhance their physicochemical properties, including solubility and dissolution rate (Dwichandra Putra et al., 2018; Yuliandra et al., 2019), compressibility (Rahman et al., 2012; Paramanandana et al., 2020), physical and chemical stability (Vangala et al., 2011; Sopyan et al., 2017), bioavailability, and pharmacological effectiveness (Shete et al., 2015; Yuliandra et al., 2018). Multi-component crystals of TMP have been extensively investigated, including a cocrystal with sulfamethoxazole and salts of trimethoprim with cinnamic acid, acetic acid, formic acid, maleic acid, and mefenamic acid (Bryan et al., 1987; Prabakaran et al., 2001; Umadevi et al., 2002; Muthiah et al., 2006; Zaini et al., 2017; Bhattacharya et al., 2020). However, to the best of our knowledge, there are no reports on the formation of multi-component crystals of TMP with malic acid (MA) nor an investigation into its physicochemical properties. MA (Figure 1B) is a dicarboxylate acid derivative used for flavouring food and as a pharmaceutical agent. MA is currently recognised as a safe excipient by the Food and Drug Administration (Ober et al., 2013).

(A)

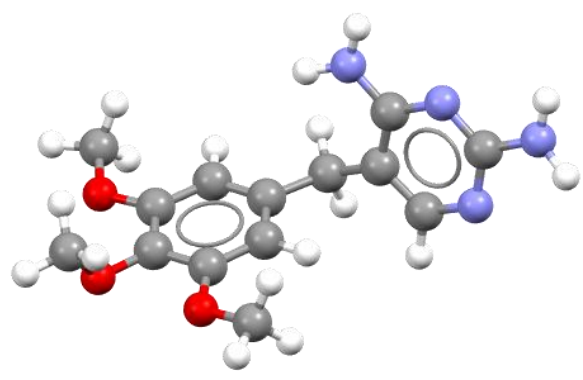

(B)

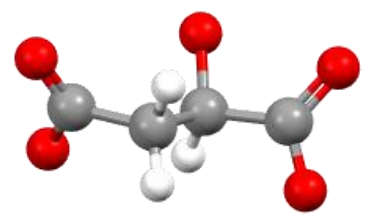

Figure 1: 3D structures of A) trimethoprim and B) malic acid
This study sought to prepare multi-component drug crystals of TMP and MA via solvent co-evaporation. The solid-state properties of the multi-component crystals were characterised by scanning electron microscopy (SEM), powder X-ray diffraction (PXRD), differential thermal analysis (DTA) for thermal behaviour, and Fourier transform infrared (FT-IR) spectroscopy. The solubility and antibacterial activities of the TMP-MA multi-component crystals were evaluated and compared to that of TMP alone.

\section{Materials and methods}

\section{Materials}

TMP was obtained from PT. Waris (Jakarta, Indonesia; Imported from Shouguang Fukang Pharmaceutical Co., Ltd., batch no. A-20111307093). MA, methanol p.a., sodium dihydrogen phosphate, nutrient agar (NA), and HPLC grade acetonitrile were purchased from Merck KGaA (Darmstadt, Germany). Sodium chloride (0.9\%) was obtained from Otsuka Pharmaceutical Co., Ltd. (Tokyo, Japan). Ethyl alcohol (70 \%) and distilled water were purchased from Brataco (Jakarta, Indonesia).

\section{Preparation of multi-component crystals of TMP-MA}

Equimolar TMP (0.01 mol, $2.9 \mathrm{~g}$ ) and MA (0.01 mol, 1.37 g) were combined in a glass beaker, dissolved in methanol and stirred to form a clear solution. The solvent was evaporated at an ambient temperature for 48h. The TMP-MA multi-component crystals were stored in a desiccator for further characterisation.

\section{Characterisation of solid-state properties}

Powder X-ray diffraction analysis

PXRD analysis was performed using an X-ray diffractometer with monochromatic CuK $\alpha$ (PANalytical Inc., Almelo, The Netherlands) and a generator voltage and current of $40 \mathrm{kV}$ and $30 \mathrm{~mA}$, respectively. PXRD patterns were recorded from $10-40^{\circ}$ on the $2 \theta$ at a step size of $0.02^{\circ}$.

\section{Differential thermal analysis}

Thermograms of TMP, MA, and the multi-component crystal were obtained using an FP85 TA Cell differential thermal analyser (Mettler Toledo, Greifensee, Switzerland). Samples ( 2-3 $\mathrm{mg}$ ) were placed in aluminium pans, sealed, and heated from $50-250{ }^{\circ} \mathrm{C}$ at a rate of $10^{\circ} \mathrm{C} / \mathrm{min}$. 


\section{Fourier transform infrared spectroscopy analysis}

FT-IR spectra of TMP and the TMP-MA multicomponent crystal were obtained using a 1600 Series FT-IR spectrometer (PerkinElmer, Rodgau, Germany). Samples ( 2-3 $\mathrm{mg}$ ) were mixed with dry potassium bromide and scanned from $4000-400 \mathrm{~cm}^{-1}$.

\section{Scanning electron microscopy analysis}

The crystal habits of TMP, MA, and the multicomponent crystal were investigated by SEM (JSM6360LA, JEOL, Ltd., Tokyo, Japan). The sample was glued onto a metal stub with double-sided adhesive tape and sputter-coated with gold/palladium ( $\mathrm{Au} / \mathrm{Pd}$ $80 / 20 \%$ ) under vacuum before analysis.

\section{Solubility test}

Excess TMP and its multi-component crystal were added to an Erlenmeyer flask containing distilled water $(100 \mathrm{~mL})$. The samples were shaken at an ambient temperature for $24 \mathrm{~h}$ using an orbital shaker. The samples were then filtered through a 0.45 -micron membrane filter, diluted with the mobile phase, and injected into an HPLC column (Hitachi, Ltd., Tokyo, Japan) with a flow rate of $1 \mathrm{~mL} / \mathrm{min}$ to measure the concentrations. Acetonitrile and sodium dihydrogen phosphate $(\mathrm{pH}=3)$ with orthophosphate acid (70:30) were used as the mobile phase. TMP was detected by UV light at a wavelength of $287 \mathrm{~nm}$. The retention time $\left(t_{R}\right)$ of TMP was $1.4 \mathrm{~min}$. All experiments were conducted in triplicate.

\section{In vitro antibacterial activity study}

The antibacterial activity of TMP and the TMP-MA multi-component crystal against Escherichia coli as a model bacterial species was investigated. NA medium was prepared ( $20 \mathrm{~g}$ in $1 \mathrm{~L}$ distilled water) and sterilised in an autoclave at $121^{\circ} \mathrm{C}$ and 2 atm for $15 \mathrm{~min}$. Bacteria were cultured at $37^{\circ} \mathrm{C}$ and incubated for $24 \mathrm{~h}$. Bacterial colonies were swabbed, suspended in normal saline solution, vortexed, and incubated for two hours until the turbidity of a McFarland standard was achieved ( 3 $x 10^{8} \mathrm{CFU} / \mathrm{mL}$ ). Antimicrobial activity assays were performed using the agar disk diffusion method with incubation at $37^{\circ} \mathrm{C}$ for $24 \mathrm{~h}$. Test compounds were prepared in multiple concentrations $(1,5,10$, and 20 $\%)$, and the diameters of the zones of inhibition were measured. All assays were performed in triplicate.

\section{Data analysis}

The data from the experiment are presented as mean \pm SD. Statistical analysis for the diameter of inhibition before and after the formation of multi-component crystals was performed by using a paired t-test. The significance level was taken at a 95\% confidence interval. The analysis was carried out using IBM SPSS Statistics version 26 (IBM, New York, USA).

\section{Results}

\section{Solid-state characterisation}

PXRD patterns were obtained for TMP, MA, and the multi-component crystal phase of TMP and MA (Figure 2). TMP displays specific diffraction peaks at $2 \theta=$ $11.81,15.14,15.94,17.40,17.87,18.18,22.21,22.47$, $28.08,30.74$, and $33.44^{\circ}$. This indicated that the solid phase had a high degree of crystallinity. MA also demonstrates highly characteristic peaks at $2 \theta=16.70$, 17.16, 28.08, and $32.14^{\circ}$. The TMP-MA multicomponent crystal shows a unique PXRD pattern, lacking the diffraction peaks of isolated TMP and MA and displaying new peaks at $2 \theta=11.47,12.30,14.20$, $18.72,20.91,23.07,25.09,27.07$, and $29.02^{\circ}$.

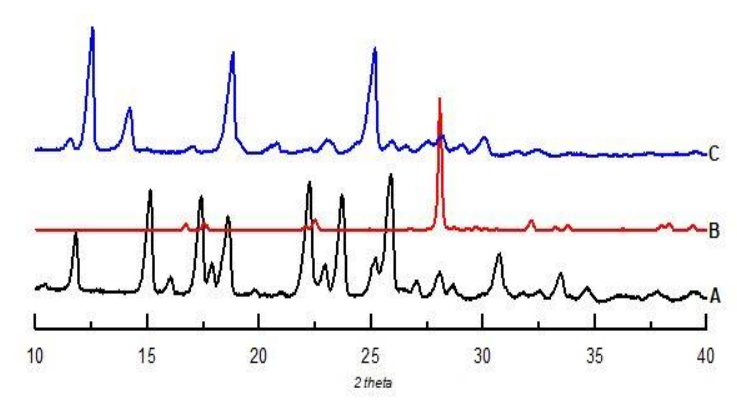

Figure 2: PXRD patterns of A) trimethoprim, B) malic acid, and C) the multi-component crystal phase of trimethoprim and malic acid

The thermodynamic behaviour of TMP, MA, and the multi-component crystal phase of TMP-MA is shown in Figure 3. The TMP thermogram exhibits a single, sharp endothermic peak at $203.4{ }^{\circ} \mathrm{C}$, corresponding to the melting point of solid TMP. MA display a melting point of $143.5^{\circ} \mathrm{C}$, followed by thermal decomposition and evaporation above $180{ }^{\circ} \mathrm{C}$. The DTA thermogram of the TMP-MA multi-component crystal is noticeably different from the starting materials (Figure $3 \mathrm{C}$ ). A unique endothermic peak of the multi-component crystal can be seen at $212.5^{\circ} \mathrm{C}$. Meanwhile, no thermal events occurred before the melting point of the multicomponent crystal. 


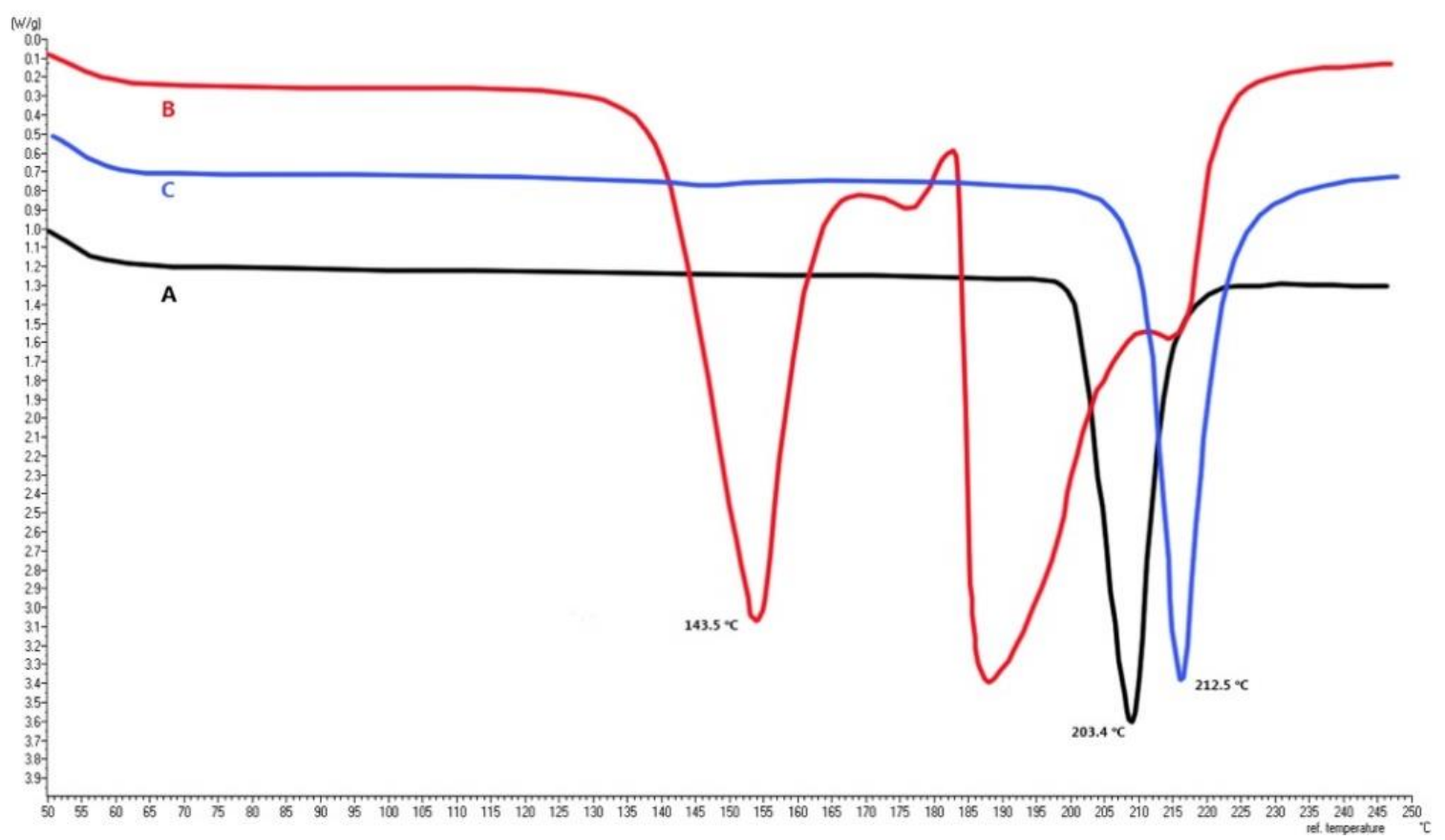

Figure 3: DTA thermograms of A) trimethoprim, B) malic acid, and C) the multi-component crystal phase of trimethoprim and malic acid

The FT-IR spectrum of TMP (Figure $4 \mathrm{~A}$ ) shows $\mathrm{N}-\mathrm{H}$ stretching frequencies at 3470.15 and $3318.25 \mathrm{~cm}^{-1}$ and an $\mathrm{N}-\mathrm{H}$ bending mode at $1632.86 \mathrm{~cm}^{-1}$. Pivotal changes in the vibrational modes were observed in the FT-IR spectra of the multi-component crystal (Figure
4B). The $\mathrm{N}-\mathrm{H}$ stretching modes shifted to 3400.56 and $3180.83 \mathrm{~cm}^{-1}$, and $\mathrm{N}-\mathrm{H}$ bending occurred at 1685.06 $\mathrm{cm}^{-1}$. The MA carboxylate anion band appears at $1590.20 \mathrm{~cm}^{-1}$, indicating proton transfer between the MA carboxylate anion and TMP.

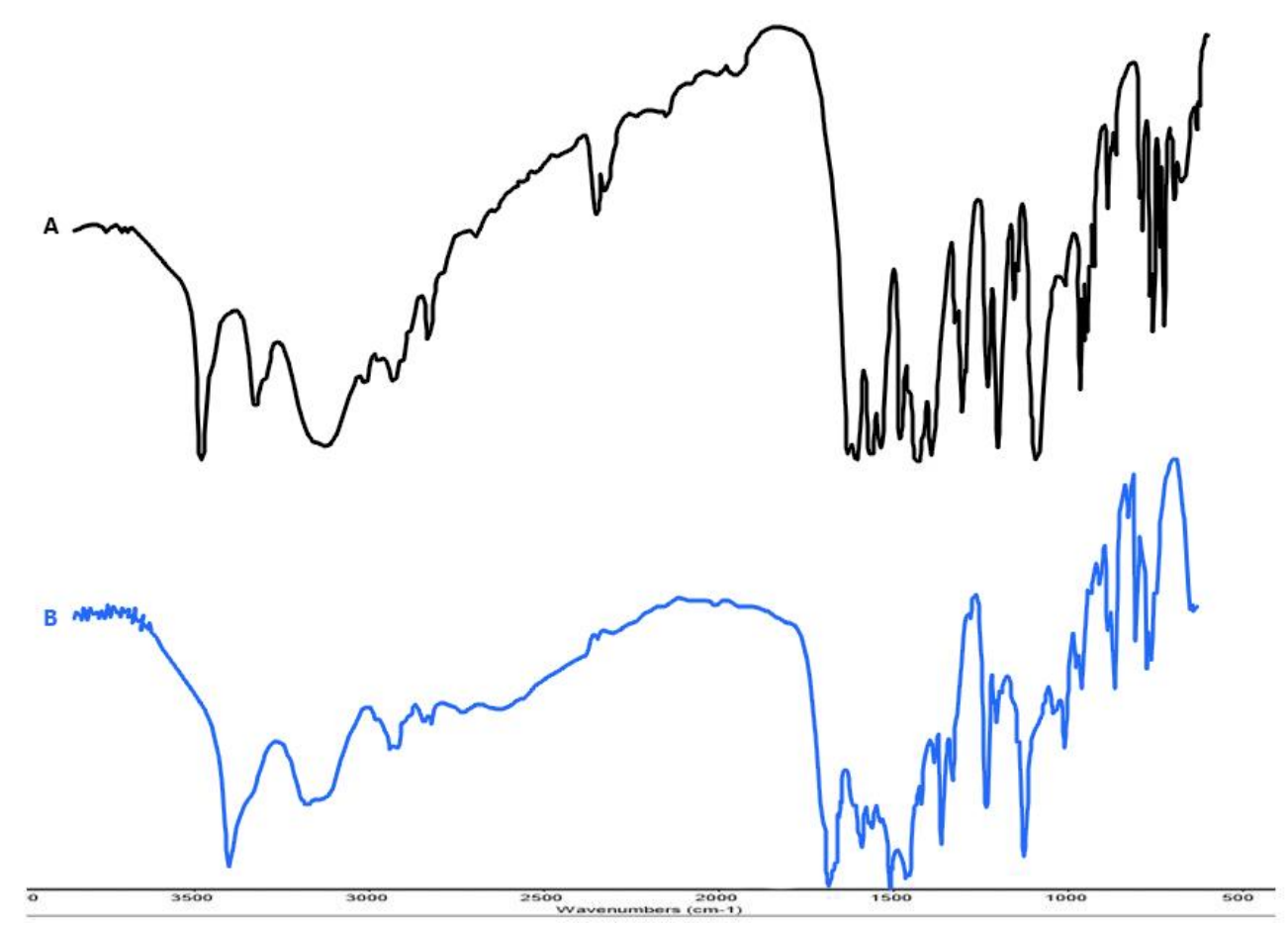

Figure 4: FT-IR spectra of A) trimethoprim and B) the multi-component crystal phase of trimethoprim and malic acid 
The crystal habits of TMP, MA, and the TMP-MA multicomponent crystal were imaged via SEM (Figure 5). TMP appears as fine, irregular-shaped particles, while MA appears as large, agglomerate-shaped particles.
The multi-component crystals of TMP-MA (Figure 5C) are significantly different from the starting materials and occur as long rod-shaped particles.
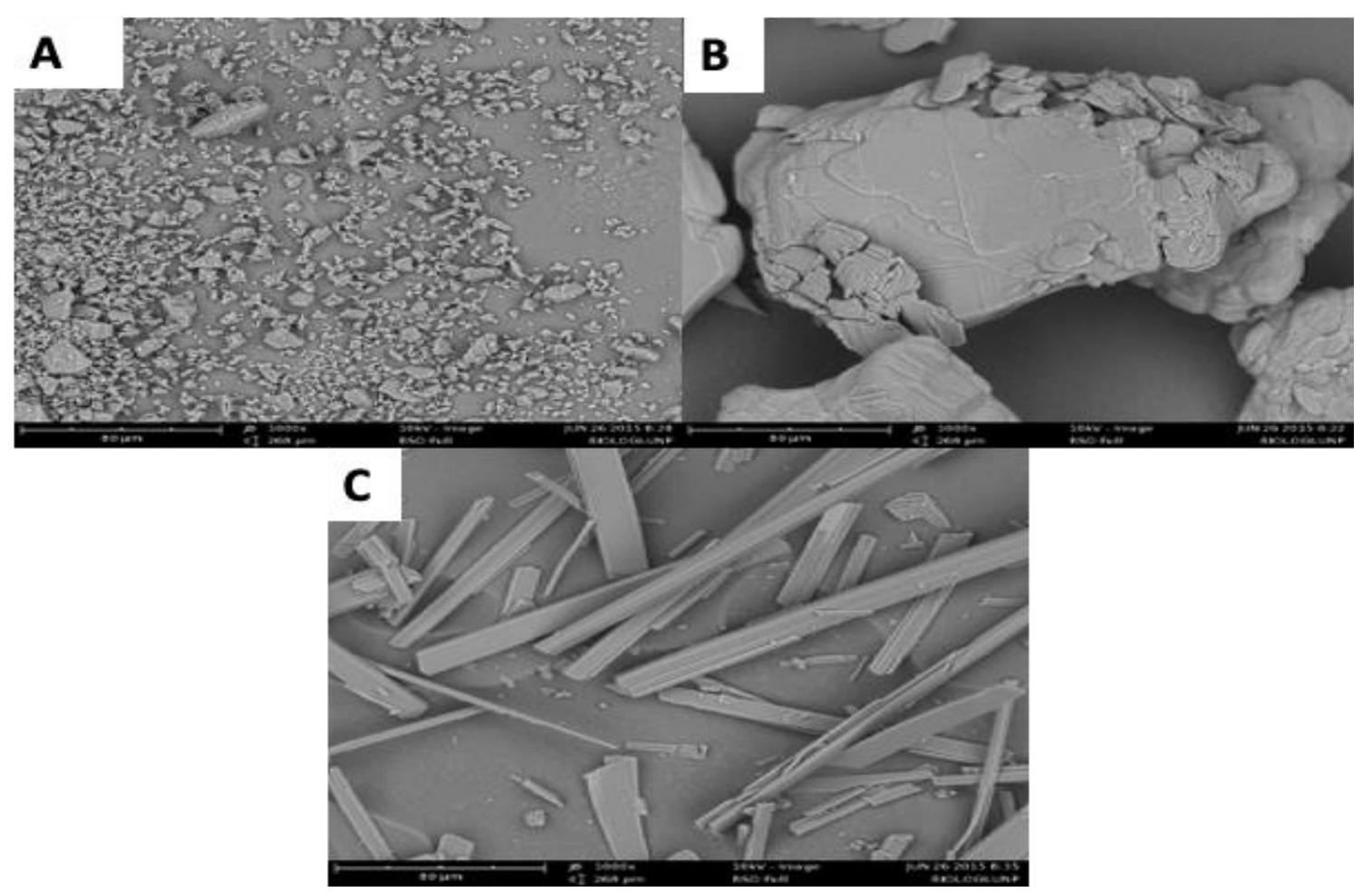

Figure 5: SEM micrographs of A) trimethoprim, B) malic acid, and C) the multi-component crystal of trimethoprim and malic acid (1000x magnification)

\section{Solubility studies}

The results of the solubility studies are presented in Table 1. The data show the multi-component crystal of TMP-MA was 2.5-fold more soluble than TMP alone.

Table 1: Solubility of trimethoprim (TMP) and its multicomponent crystal with malic acid (TMP-MA) $(n=6)$.

\begin{tabular}{ll}
\hline Compound & Solubility \pm SD $(\mathrm{mcg} / \mathrm{mL})$ \\
\hline TMP & $19.04 \pm 0.05$ \\
TMP-MA & $47.43 \pm 0.07$
\end{tabular}

\section{In vitro antibacterial activity study}

Treatment with TMP-MA multi-component crystals resulted in a notably larger (22\%) zone of inhibition than TMP alone (Figure 6). Importantly, this increase was obtained for every concentration tested. The statistical analysis showed a significant increase in the inhibition zone after the formation of multi-component crystals of TMP-MA $(p=0.01)$.

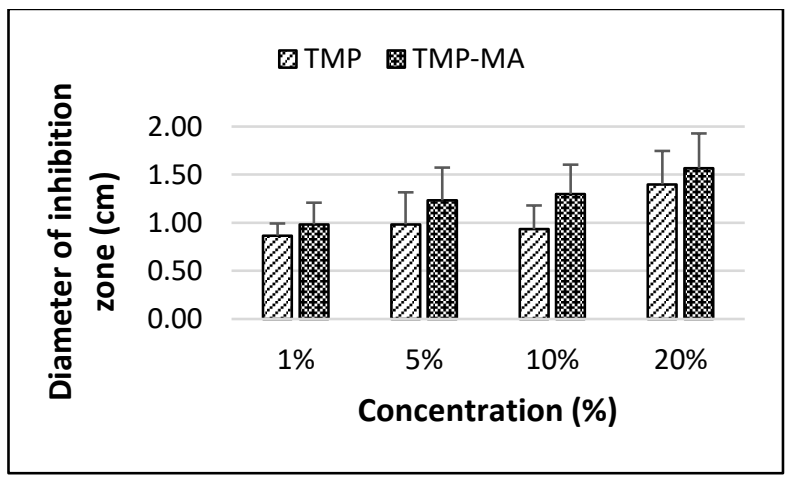

Paired t-test showed a significant increase in the inhibition zone after the formation of multi-component crystal of TMP-MA $(p<0.05)$

Figure 6: Antibacterial activity of trimethoprim (TMP) and trimethoprim-malic acid multi-component crystals (TMP-MA) against $E$. coli $(n=24)$ 


\section{Discussion}

Poor aqueous solubility has been the major obstacle in the development of drugs, especially in the path of drug delivery. It makes it difficult for the sufficient concentration of drug molecules to be delivered at their sites of action. This issue has become more important as oral ingestion is currently the most common and convenient route of drug administration (Savjani et al., 2012). The formation of multicomponent crystals is a promising approach to enhance the solubility of poorly water-soluble drugs such as trimethoprim (TMP). The present study aimed to prepare the multi-component crystals of TMP with malic acid (MA) and study their solubility and in vitro antibacterial activity.

PXRD is a reliable technique for characterising the solidstate properties of active pharmaceutical ingredients. PXRD patterns of a solid can be considered as fingerprints of the crystal phases; therefore, it can confirm the formation of new crystalline phases through the intermolecular interactions between two solid phases. A multi-component crystal phase is formed if the PXRD pattern of the binary mixture is distinct from its starting components (Karagianni et al., 2018; Thakral et al., 2018). The PXRD pattern of the present study (Figure 2) showed specific diffraction peaks of TMP, which are similar to a previous report (Zaini et al., 2017). The unique pattern of TMP-MA, which established new peaks, supports the formation of a distinct crystalline phase composed of both TMP and MA. The formation of a multi-component crystal of an active pharmaceutical ingredient and coformer (salttype or cocrystal) can be predicted using the $\Delta$ pKa rule. If the difference in pKa between the active pharmaceutical ingredients and coformers is $\geq 3$ then the binary system tends to form a salt-type multicomponent crystal, whereas a cocrystal form if $\Delta \mathrm{pKa}<$ 3 (Cruz-Cabeza, 2012; Loya et al., 2019). TMP is a weak base (pKa $=7.3$ ), while MA is a weak dicarboxylic acid (pKa = 3.4) (Molu \& Yurdakoç, 2010; Uslu \& Kırbaşlar, 2010). The $\triangle p K a$ for TMP and MA = 3.9, which suggests that the binary system will assume a salt-type multicomponent crystal phase.

Thermal analysis is a simple and rapid method for determining the formation of a multi-component crystal phase (Lu et al., 2008). DTA was used to evaluate the thermodynamic behaviour of intact materials and their multi-component crystal phase. The melting point of MA and its thermal decomposition and evaporation above $180{ }^{\circ} \mathrm{C}$ are in agreement with previous work (Fernandes et al., 2019). On the other hand, the unique endothermic peak of the multi-component crystal $\left(212.5^{\circ} \mathrm{C}\right)$ indicates the formation of a new solid phase. The absence of thermal events before the melting point of the multi-component crystal suggests that it was either non-solvate or anhydrous. These results are also supported by the PXRD analysis.

FT-IR spectroscopy is an important method for confirming the formation of multi-component crystals. Changes in vibrational mode energies can suggest intramolecular interactions between the functional groups of the active pharmaceutical ingredients and excipients (da Silva et al., 2016). The FT-IR spectrum of TMP (Figure 4A) showed similar properties with a previous study (Bettinetti et al., 1983). MA is an organic acid (dicarboxylate acid) containing several carboxylate and hydroxyl functional groups, which act as proper coformers, interacting with target functional groups of the drug (a weak base) through non-covalent interactions, such as hydrogen and van der Waals bonds (Chadha et al., 2016; Cugovčan et al., 2017). TMP and MA interact via $\mathrm{N}-\mathrm{H} \cdots \cdots . . \mathrm{O}$ hydrogen bonding between the TMP cation's protonated pyrimidine moiety and MA carboxylate anion. Previous studies have elucidated the structures of the multi-component crystal phase of TMP with various organic acids (Bettinetti et al., 1983; Bryan et al., 1987; Muthiah et al., 2006; Prabakaran et al., 2001; Umadevi et al., 2002). The intermolecular interaction pattern is similar to our finding.

Co-crystallisation of multi-component crystals from organic solvent can produce a crystal habit differing from its starting materials. The crystal habit is affected by various factors, including solvent type, evaporation temperature, and degree of supersaturation. A change of crystal habit during multi-component crystal formation can influence the dissolution rate, flowability, and mechanical properties of active pharmaceutical ingredients (Serrano et al., 2016; Sathisaran \& Dalvi, 2018). The crystal habits of TMP, MA, and the TMP-MA multi-component crystal were imaged via SEM (Figure 5). The new crystal habit seen at the multi-component crystals of TMP-MA (Figure $5 \mathrm{C}$ ) is a strong indicator of the formation of a multicomponent crystal phase (Basavoju et al., 2008).

Solubility plays a significant role in the gastrointestinal absorption of orally administered pharmaceutical agents. Drugs that are poorly soluble in water generally have limited bioavailability in the systemic circulation. Modifying the solid-state properties of active pharmaceutical ingredients through the formation of multi-component crystals with suitable coformers is a promising technique to improve solubility and pharmacological effectiveness (Kawabata et al., 2011; Berry \& Steed, 2017). The primary advantage of this approach is the ability to maintain the drug in a solid crystalline phase and sustain its thermodynamic stability. In addition, this technique does not change the pharmacophore structure of the active 
pharmaceutical ingredients (Dwichandra Putra et al., 2018).

The present solubility studies showed that the multicomponent crystal of TMP-MA was 2.5-fold more soluble than TMP alone. Several mechanisms can explain the improved solubility of TMP in the multicomponent crystal. For example, the incorporation of soluble excipients as coformers in the multi-component crystal phase can increase the solubility of poorly soluble drugs. In addition, the formation of a salt-type multi-component crystal of TMP-MA improves solubility as a result of a higher affinity in water. Salttype multi-component crystals immediately dissociate in an aqueous medium to cations and anions (Dwichandra Putra et al., 2016; Yuliandra et al., 2019; Zaini et al., 2019).

Agar disk diffusion was used to determine whether the TMP-MA multi-component crystals displayed increased antibacterial activity compared to TMP alone. This technique benefits from simplicity, affordability, ability to test against a wide number of bacterial species, and ease of analysis (Balouiri et al., 2016). The present study showed a $22 \%$ larger inhibition zone of multicomponent crystal TMP-MA as compared with the intact TMP. Some recent studies also report the improvement of antibacterial activities through the formation of multi-component crystals of antibacterial agents. For example, the minimum inhibitory concentration of sulfaguanidine against $E$. coli is improved from 35 to $25 \mu \mathrm{g} / \mathrm{ml}$ after its cocrystal formation with thiobarbituric acid (Abidi et al., 2018). Sulfamethazine, another antibacterial agent from the sulfonamide class, shows a two-fold higher inhibition rate against the same bacterial species when prepared as a cocrystal with $p$-aminobenzoic acid (Pan et al., 2019). A previous study also finds similar results for this antibacterial agent when cocrystallised with aminosalicylic acid (Serrano et al., 2016). These results suggest that the formation of multi-component crystals is a propitious strategy to improve the efficacy of drugs, such as antibacterial agents, with poor aqueous solubility.

\section{Conclusions}

This study shows that the formation of a multicomponent crystal phase between TMP and MA successfully improves the solubility and antibacterial activity of TMP. The solid-state properties, including the PXRD pattern, thermal behaviour, and crystal habits of the multi-component crystal of TMP-MA are remarkably different from that of TMP alone. Meanwhile, the formation of multi-component crystals of TMP-MA significantly improves the antibacterial activity in vitro.

\section{References}

Abidi, S. S. A., Azim, Y., Khan, S. N., \& Khan, A. U. (2018). Sulfaguanidine cocrystals: Synthesis, structural characterisation and their antibacterial and hemolytic analysis. Journal of Pharmaceutical and Biomedical Analysis, 149, 351-357. https://doi.org/10.1016/j.jpba.2017.11.028

Balouiri, M., Sadiki, M., \& Ibnsouda, S. K. (2016). Methods for in vitro evaluating antimicrobial activity: A review. Journal of Pharmaceutical Analysis, 6(2), 71-79. https://doi.org/10.1016/j.jpha.2015.11.005

Basavoju, S., Boström, D., \& Velaga, S. P. (2008). IndomethacinSaccharin Cocrystal: Design, Synthesis and Preliminary Pharmaceutical Characterization. Pharmaceutical Research, 25(3), 530-541. https://doi.org/10.1007/s11095-007-9394-1

Berry, D. J., \& Steed, J. W. (2017). Pharmaceutical cocrystals, salts and multi-component systems; intermolecular interactions and property based design. Advanced Drug Delivery Reviews, 117, 324. https://doi.org/10.1016/j.addr.2017.03.003

Bettinetti, G. P., Caramella, C., Giordano, F., La Manna, A., Margheritis, C., \& Sinistri, C. (1983). Thermal analysis of binary systems of the pharmaceuticals trimethoprim and benzoic acid. Journal of Thermal Analysis, 28(2), 285-293. https://doi.org/10.1007/BF01983262

Bhattacharya, B., Das, S., Lal, G., Soni, S. R., Ghosh, A., Reddy, C. M., \& Ghosh, S. (2020). Screening, crystal structures and solubility studies of a series of multidrug salt hydrates and cocrystals of fenamic acids with trimethoprim and sulfamethazine. Journal of Molecular Structure, 1199, 127028. https://doi.org/10.1016/j.molstruc.2019.127028

Bryan, R. F., Haltiwanger, R. C., \& Woode, M. K. (1987). Trimethoprim acetate. Acta Crystallographica Section C Crystal Structure Communications, 43(12), 2412-2415. https://doi.org/10.1107/S0108270187087584

Byrn, S. R., \& Henck, J.O. (2012). Optimising the physical form opportunities and limitations. Drug Discovery Today: Technologies, 9(2), e73-e78. https://doi.org/10.1016/j.ddtec.2012.03.006

Chadha, R., Rani, D., \& Goyal, P. (2016). Novel cocrystals of gliclazide: characterisation and evaluation. CrystEngComm, 18(13), 2275-2283. https://doi.org/10.1039/C5CE02402A

Cherukuvada, S., Kaur, R., \& Guru Row, T. N. (2016). Cocrystallization and small molecule crystal form diversity: from pharmaceutical to materials applications. CrystEngComm, 18(44), 8528-8555. https://doi.org/10.1039/C6CE01835A

Cruz-Cabeza, A. J. (2012). Acid-base crystalline complexes and the pKa rule. CrystEngComm, 14(20), 6362. https://doi.org/10.1039/c2ce26055g

Cugovčan, M., Jablan, J., Lovrić, J., Cinčić, D., Galić, N., \& Jug, M. (2017). Biopharmaceutical characterisation of praziquantel cocrystals and cyclodextrin complexes prepared by grinding. Journal of Pharmaceutical and Biomedical Analysis, 137, 42-53. https://doi.org/10.1016/j.jpba.2017.01.025 
da Silva, C. C., Guimarães, F. F., Ribeiro, L., \& Martins, F. T. (2016). Salt or cocrystal of salt? Probing the nature of multi-component crystal forms with infrared spectroscopy. Spectrochimica Acta Part A: Molecular and Biomolecular Spectroscopy, 167, 89-95. https://doi.org/10.1016/j.saa.2016.05.042

Dwichandra Putra, O., Umeda, D., Fujita, E., Haraguchi, T., Uchida, T., Yonemochi, E., \& Uekusa, H. (2018). Solubility improvement of benexate through salt formation using artificial sweetener. Pharmaceutics, 10(2), 64. https://doi.org/10.3390/pharmaceutics10020064

Dwichandra Putra, O., Yonemochi, E., \& Uekusa, H. (2016). Isostructural Multicomponent Gliclazide Crystals with Improved Solubility. Crystal Growth \& Design, 16(11), 65686573. https://doi.org/10.1021/acs.cgd.6b01279

Fernandes, R. P., do Nascimento, A. L. C. S., Carvalho, A. C. S., Teixeira, J. A., Ionashiro, M., \& Caires, F. J. (2019). Mechanochemical synthesis, characterisation, and thermal behavior of meloxicam cocrystals with salicylic acid, fumaric acid, and malic acid. Journal of Thermal Analysis and Calorimetry, 138(1), 765-777. https://doi.org/10.1007/s10973-019-08118-7

Guptat, R. L., Kumar, R., \& Singla, A. K. (1991). Enhanced dissolution and absorption of trimethoprim from coprecipitates with polyethylene glycols and polyvinylpyrrolidone. Drug Development and Industrial Pharmacy, 17(3), 463-468. https://doi.org/10.3109/03639049109043840

Hawser, S., Lociuro, S., \& Islam, K. (2006). Dihydrofolate reductase inhibitors as antibacterial agents. Biochemical Pharmacology, 71(7), 941-948. https://doi.org/10.1016/j.bcp.2005.10.052

Karagianni, A., Malamatari, M., \& Kachrimanis, K. (2018). Pharmaceutical Cocrystals: New Solid Phase Modification Approaches for the Formulation of APIs. Pharmaceutics, 10(1), 18. https://doi.org/10.3390/pharmaceutics10010018

Kawabata, Y., Wada, K., Nakatani, M., Yamada, S., \& Onoue, S. (2011). Formulation design for poorly water-soluble drugs based on biopharmaceutics classification system: Basic approaches and practical applications. International Journal of Pharmaceutics, 420(1), 1-10. https://doi.org/10.1016/j.ijpharm.2011.08.032

Li, N., Zhang, Y.-H., Wu, Y.-N., Xiong, X.-L., \& Zhang, Y.-H. (2005). Inclusion complex of trimethoprim with $\beta$-cyclodextrin. Journal of Pharmaceutical and Biomedical Analysis, 39(3-4), 824-829. https://doi.org/10.1016/j.jpba.2005.05.011

Loya, J. D., Li, S. J., Unruh, D. K., \& Hutchins, K. M. (2019). Application of the pKa rule to synthesise salts of bezafibrate. Supramolecular Chemistry, 31(8), 558-564. https://doi.org/10.1080/10610278.2019.1635695

Lu, E., Rodríguez-Hornedo, N., \& Suryanarayanan, R. (2008). A rapid thermal method for cocrystal screening. CrystEngComm, 10(6), 665. https://doi.org/10.1039/b801713c

Molu, Z. B., \& Yurdakoç, K. (2010). Preparation and characterisation of aluminum pillared K10 and KSF for adsorption of trimethoprim. Microporous and Mesoporous Materials, 127(1-2), 50-60. https://doi.org/10.1016/j.micromeso.2009.06.027

Muthiah, P. T., Francis, S., Rychlewska, U., \& Warżajtis, B. (2006). Crystal engineering of analogous and homologous organic compounds: hydrogen bonding patterns in trimethoprim hydrogen phthalate and trimethoprim hydrogen adipate. Beilstein Journal of Organic Chemistry, 2(1), 8. https://doi.org/10.1186/1860-5397-2-8
Ober, C. A., Montgomery, S. E., \& Gupta, R. B. (2013). Formation of itraconazole/L-malic acid cocrystals by gas antisolvent cocrystallisation. Powder Technology, 236, 122131. https://doi.org/10.1016/j.powtec.2012.04.058

Pan, X., Zheng, Y., Chen, R., Qiu, S., Chen, Z., Rao, W., Chen, S., You, Y., Lü, J., Xu, L., \& Guan, X. (2019). Cocrystal of Sulfamethazine and pAminobenzoic Acid: Structural Establishment and Enhanced Antibacterial Properties. Crystal Growth \& Design, 19(4), 2455-2460. https://doi.org/10.1021/acs.cgd.9b00137

Paramanandana, A., Erfadrin, V. E., Sari, R., Paramita, D. P., \& Setyawan, D. (2020). Compression force effect on characteristics of loratadine-succinic acid cocrystal prepared by slurry method. Journal of Research in Pharmacy, 24(3), 410-415. https://doi.org/10.35333/jrp.2020.163

Pawar, P. H., Pawar, A. P., Mahadik, K. R., \& Paradkar, A. R. (1998). Evaluation of tableting properties of agglomerates obtained by spherical crystallisation of trimethroprim. Indian Journal of Pharmaceutical Sciences, 60(1), 24-28.

Prabakaran, P., Robert, J. J., Thomas Muthiah, P., Bocelli, G., \& Righi, L. (2001). Aminopyrimidine-carboxyl(ate) interactions in trimethoprim maleate, an antifolate drug. Acta Crystallographica Section C Crystal Structure Communications, 57(4), 459-461. https://doi.org/10.1107/\$0108270101000269

Rahman, Z., Samy, R., Sayeed, V. A., \& Khan, M. A. (2012). Physicochemical and mechanical properties of carbamazepine cocrystals with saccharin. Pharmaceutical Development and Technology, 17(4), 457-465. https://doi.org/10.3109/10837450.2010.546412

Sathisaran, I., \& Dalvi, S. (2018). Engineering Cocrystals of Poorly Water-Soluble Drugs to Enhance Dissolution in Aqueous Medium. Pharmaceutics, 10(3), 108. https://doi.org/10.3390/pharmaceutics10030108

Savjani, K. T., Gajjar, A. K., \& Savjani, J. K. (2012). Drug Solubility: Importance and Enhancement Techniques. ISRN Pharmaceutics, 2012, 195727. https://doi.org/10.5402/2012/195727

Serrano, D. R., Persoons, T., D’Arcy, D. M., Galiana, C., DeaAyuela, M. A., \& Healy, A. M. (2016). Modelling and shadowgraph imaging of cocrystal dissolution and assessment of in vitro antimicrobial activity for sulfadimidine/4-aminosalicylic acid cocrystals. European Journal of Pharmaceutical Sciences, 89, 125-136. https://doi.org/10.1016/j.ejps.2016.04.030

Serrano, D. R., O'Connell, P., Paluch, K. J., Walsh, D., \& Healy, A. M. (2016). Cocrystal habit engineering to improve drug dissolution and alter derived powder properties. Journal of Pharmacy and Pharmacology, 68(5), 665-677. https://doi.org/10.1111/jphp.12476

Shete, A., Murthy, S., Korpale, S., Yadav, A., Sajane, S., Sakhare, S., \& Doijad, R. (2015). Cocrystals of itraconazole with amino acids: Screening, synthesis, solid state characterisation, in vitro drug release and antifungal activity. Journal of Drug Delivery Science and Technology, 28, 46-55. https://doi.org/10.1016/j.jddst.2015.05.006

Sheth, A. R., \& Grant, D. J. W. (2005). Relationship between the Structure and Properties of Pharmaceutical Crystals. KONA Powder and Particle Journal, 23, 36-48. https://doi.org/10.14356/kona.2005008 
Sopyan, I., Fudholi, A., Muchtaridi, M., \& Sari, I. P. (2017). Simvastatin-nicotinamide cocrystal: design, preparation and preliminary characterisation. Tropical Journal of Pharmaceutical Research, 16(2), 297. https://doi.org/10.4314/tjpr.v16i2.6

Thakral, N. K., Zanon, R. L., Kelly, R. C., \& Thakral, S. (2018). Applications of Powder X-Ray Diffraction in Small Molecule Pharmaceuticals: Achievements and Aspirations. Journal of Pharmaceutical Sciences, 107(12), 2969-2982. https://doi.org/10.1016/j.xphs.2018.08.010

Umadevi, B., Prabakaran, P., \& Muthiah, P. T. (2002). A pseudoquadruple hydrogen-bonding motif consisting of six $\mathrm{N}-\mathrm{H}$...O hydrogen bonds in trimethoprim formate. Acta Crystallographica Section C Crystal Structure Communications, 58(8), 0510-0512. https://doi.org/10.1107/S0108270102011150

Uslu, H., \& Kırbaşlar, Ş. ì. (2010). Extraction of aqueous of malic acid by trioctylamine extractant in various diluents. Fluid Phase Equilibria, 287(2), 134-140. https://doi.org/10.1016/j.fluid.2009.09.022

Vangala, V. R., Chow, P. S., \& Tan, R. B. H. (2011). Characterisation, physicochemical and photo-stability of a cocrystal involving an antibiotic drug, nitrofurantoin, and 4-hydroxybenzoic acid. CrystEngComm. https://doi.org/10.1039/c0ce00772b

Yuliandra, Y., Izadihari, R., Rosaini, H., \& Zaini, E. (2019). Multicomponent crystals of mefenamic acid - tromethamine with improved dissolution rate. Journal of Research in Pharmacy, 23(6), 988-996. https://doi.org/10.35333/jrp.2019.63

Yuliandra, Y., Zaini, E., Syofyan, S., Pratiwi, W., Putri, L. N., Pratiwi, Y. S., \& Arifin, H. (2018). Cocrystal of ibuprofennicotinamide: Solid-state characterisation and in vivo analgesic activity evaluation. Scientia Pharmaceutica, 86(2), 23. https://doi.org/10.3390/scipharm 86020023

Zaini, E., Fitriani, L., Sari, R. Y., Rosaini, H., Horikawa, A., \& Uekusa, H. (2019). Multi-component Crystal of Mefenamic Acid and NMethyl-d-Glucamine: Crystal Structures and Dissolution Study. Journal of Pharmaceutical Sciences, 108(7), 2341-2348. https://doi.org/10.1016/j.xphs.2019.02.003

Zaini, E., Sumirtapura, Y. C., Halim, A., Fitriani, L., \& Soewandhi, S. N. (2017). Formation and characterisation of sulfamethoxazole-trimethoprim cocrystal by milling process. Journal of Applied Pharmaceutical Science, 7(12), 169-173. https://doi.org/10.7324/JAPS.2017.71224 